\title{
NOVEL GENOTYPE IN TWO SIBLINGS WITH 5- $\alpha-$ REDUCTASE 2 DEFICIENCY: DIFFERENT CLINICAL COURSE DUE TO THE TIME OF DIAGNOSIS
}

\author{
Kocova $\mathrm{M}^{1, *}$, Plaseska-Karanfilska $\mathrm{D}^{2}$, Noveski $\mathrm{P}^{2}$, Kuzmanovska $\mathrm{M}^{2}$ \\ *Corresponding Author: Professor Dr. Mirjana Kocova, University Pediatric Clinic, Department of \\ Endocrinology \& Genetics, M. Tereza 17, Skopje 1000, Republic of North Macedonia. Tel: +389-70- \\ 242-694. Fax: +389-317-6167. E-mail: mirjanakocova@yahoo.com
}

\begin{abstract}
Steroid 5- $\alpha$-reductase-2 (5-ARD) deficiency is a result of mutations of the $S R D 5 A 2$ gene. It causes the disorder of sexual differentiation (DSD) in 46,XY individuals with a variable genital phenotype. We present two siblings with female external genitalia at birth and bilateral inguinal testes, raised as females. These are the first molecularly characterized patients from the Republic of North Macedonia (RN Macedonia) with a different clinical course due to the time of the diagnosis. Diagnosis of Patient 1 was based upon the detection of bilateral inguinal testes and testosterone/dihidrotestosterone ratio. Sex reversal was initiated by testes removal at the age of 20 months. Breast implantation and vaginoplasty were performed in adolescence and the girl is comfortable with the female sex. Her sibling, Patient 2, raised as a girl, was clinically assessed at 11.5 years due to the growth of phalus, deep voice and Adam's apple enlargement. No change of gender was accepted. Complex molecular analysis including multiplex quantitative fluorescent polymerase chain reaction (PCR) screening for sex chromosome aneuploidies and $S R Y$ presence, Sanger sequencing combined with multiplex ligation-dependent probe amplification (MLPA), microarraybased comparative genomic hybridization (aCGH), and real-time PCR analysis for detection of exon copy number changes confirmed a novel c. 146C $>$ A (p.Ala49Asp) point mutation in the first exon inherited from the mother, and complete deletion of the first exon and adjacent regions inherited from the father. Novel genotype causing 5-ARD is presented. Genetic analysis is useful for the diagnosis
\end{abstract}

\footnotetext{
${ }^{1}$ University Pediatric Clinic, Skopje, Republic of North Macedonia

${ }^{2}$ Research Center for Genetic Engineering and Biotechnology "Georgi D. Efremov," Macedonian Academy of Sciences and Arts, Skopje, Republic of North Macedonia
}

and timely gender assignment in patients with 5-ARD. However, final gender assignment is difficult and requires combined medical interventions.

Keywords: Disorder of sexual differentiation (DSD), 5- $\alpha$-reductase-2 (5-ARD) deficiency; SRD5A2 gene; Gender assignment; Gene deletion.

Background. Steroid 5- $\alpha$-reductase type 2 (5-ARD) deficiency is a rare autosomal recessive genetic condition causing a 46,XY disorder of sexual differentiation (DSD) with unknown incidence [1]. Patients affected by 5-ARD cannot convert testosterone to dihydrotestosterone (DHT), which is a more potent androgen than testosterone and is responsible for male external genitalia phenotype formation. Furthermore, these patients present with under virilized external genitalia. The genital phenotype can vary greatly, from complete female external genitalia on the one hand to overt male pesudohermaphroditism on the other [1-3]. The testes are normal and usually located along the inguinal canal, but can rarely be located in the abdomen or in the scrotum. Internal genitalia are male except for the hypoplastic prostate. Occasionally, patients lack a separate vaginal opening, may have penile hypospadias or even a penile urethra. Due to the appearance of the external genitalia, these individuals are usually considered females at birth. Dihydrotestosterone deficiency is a result of recessive, mostly missense mutations of the steroid 5 - $\alpha$-reductase 2 (SRD5A2) gene located on band 2p23. The SRD5A2 gene consists of five exons and is expressed in the perineal skin and fibroblasts causing virilization in the XY fetus. The first exon of SRD5A2 is a common hotspot for mutations, however, about a dozen different mutations have been described, also involving other exons of the SRD5A2 gene $[4,5]$. Although the incidence of this disorder has not been established, it is reported with increased prevalence in parts of the world where consanguinity is more frequent in some 
kindreds (Turkey, Dominican Republic) [6-9]. The sex of rearing of affected individuals varies; both female and male sex have been chosen with a variable outcome. It is worth mentioning that during puberty some virilization appears spontaneously, even if incomplete. A different sex of rearing has been selected in affected individuals even within the same family. The success of functional sex formation and resultant quality of life is variable $[2,10]$.

Here we present the first two molecularly characterized patients with 5-ARD from the Republic of North Macedonia (RN Macedonia), both reared as females, carrying a novel compound heterozygous genotype of SRD5A2, who encountered different problems during growth and development due to the different timing of diagnosis and therapeutic approach.

Case Presentations. During this study we complied with the World Medical Association Declaration of Helsinki regarding ethical conduct of research involving human subjects. Informed consent for analyses and data presentation from the parents and Patient 1, and assent from Patient 2, was obtained. The procedures and data analysis were approved by the Institutional Ethics Committee on human research at the University Pediatric Clinic, Skopje, RN Macedonia.

Patient 1 was the second child of young, unrelated parents, born at term with a birth weight of 3400 gr and length of $50 \mathrm{~cm}$. Sexual ambiguity was noticed at birth: rugged large labia resembling scrota with $1 \mathrm{~mL}$ formation in the middle part of the inguinal channel bilaterally, a phallus of $10 \mathrm{~mm}$ in length, and an opening for putative perineal sinus urogenitalis. Blood counts and electrolytes were in the normal range. U1trasound examination of the labia showed potential testicular tissue $8 \times 10 \mathrm{~mm}$ (right) and $6 \times 10 \mathrm{~mm}$ (left). Cytogenetic analysis confirmed a 46,XY karyotype, leading to a clinical diagnosis of disorder of DSD. Urethrocystography showed a male urethra opening in the common sinus urogenitalis. Ultrasound confirmed normal kidneys, ureters and bladder, as well as adrenal glands, and no female internal genitalia in the small pelvis. The testosterone/DHT ratio was 22 suggesting 5-ARD. The girl was followed-up during the first 2 years at 3-month intervals. The parents consulted endocrinologists and psychologists about the possibilities of sex of rearing. Their decision for female sex of rearing was definite. Orchidectomy together with clitoroplasty was performed at the age of 20 months. Hormone levels [17OH progesterone (17OHP), adrenocortiotropic hormone (ACTH), Cortisol, dehydroepiandrosterone sulfate (DHEAS), follicle-stimulating hormone (FSH), luteinizing hormone (LH), thyroxine (T4) and thyroid-stimutating hormone (TSH)], measured yearly afterwards were within the normal range. At the age of 13 years hormonal analyses showed very high $\mathrm{LH}(31.7 \mathrm{mIU} / \mathrm{ml})$ and FSH $(88.5 \mathrm{mIU} / \mathrm{mL})$ levels, and undetectable estrogen.
Her bone age was 10 years, although her height was at the 50 th percentile on the growth curve. Induction of puberty with estrogen patches and contraceptives afterwards did not improve the lack of breasts. Pubic and armpit hair was scarce. Breast implants and vaginoplasty were performed at the age of 20 and 22 years, respectively, completing the female sex phenotype [Figure 1(A) and 1(B)].

Patient 2 was born as the third child in the same family with a birth weight of $3000 \mathrm{gr}$ and length of $50 \mathrm{~cm}$. No specific consultations were sought by the parents despite the advice to bring her early due to the diagnosis in her sibling till the age of 7 years when she was brought for a check-up of the genitalia. She had a normal height and weight, but was pretty muscular. Her clitoris was enlarged to $18 \mathrm{~mm}$, along with labial fusion. Other findings were normal. Her karyotype was 46,XY. The FSH, LH, DHEAS, testosterone and estrogen levels were normal. Testes were detected in the labial folds by ultrasound. Parents refused further consultations up to the age of 11.5 years when she was admitted due to a significant virilization: a deep voice, growth of Adam's apple, and growth of the phallus. Puberty stage was M1, P1-2, A1; testes were palpable in the inguinal channels bilaterally, phallus was $4 \mathrm{~cm}$ long accompanied by a perineal hypospadia and complete labial fusion [Figure 1(C) and 1(D)]. The same was documented on urethro-cystography. Pelvic ultrasound examination showed no Müllerian structures, and developed testicles $(25 \times 15 \mathrm{~mm})$ in the upper inguinal channel. Gonadotropins were high, FSH reaching levels of $60 \mathrm{mIU} / \mathrm{mL}$ and LH $22 \mathrm{mIU} / \mathrm{mL}$. The testosterone/ DHT ratio was 20 and although molecular analysis was not possible at that time, the diagnosis of 5-ARD was made. The patient had been reared as a girl since birth and had a female sex identity. The decision of the adolescent and the parents did not change after numerous consultations.

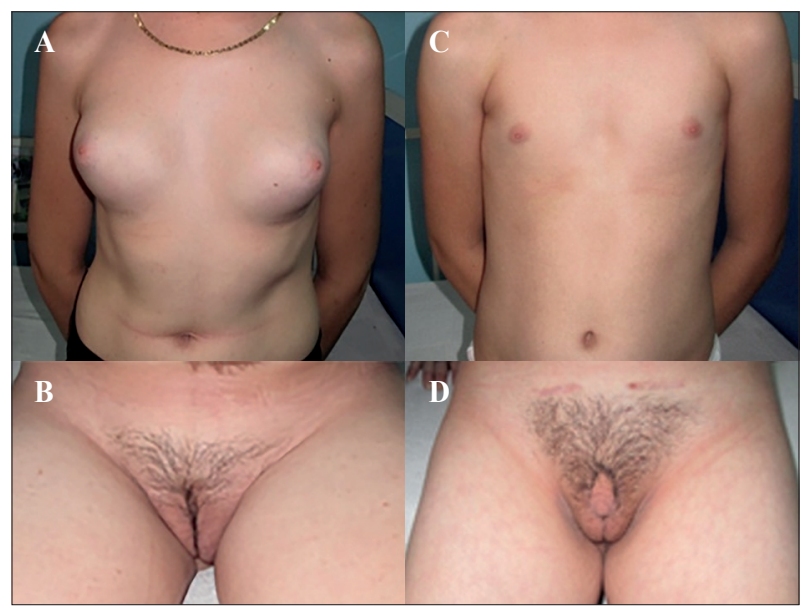

Figure 1. Physical appearance of patients. A and B) implanted breasts and genital appearance in Patient 1 at age 21.

C and D) muscular body and genital appearance of Patient 2 before surgery. 
Testes removal was performed after written consent and institutional ethical approval together with clitoroplasty. The last checkup at 13.5 years showed decrease in the size of Adam's apple and slight voice improvement.

Methods for the Genetic Analyses. Sanger sequencing of exons and exons/introns boundaries of the SRD5A2 gene was performed. Multiple ligation-dependent probe analysis (MLPA) was performed using P334-A3 Gonadal Development Disorder kit (MRC-Holland, Amsterdam, The Netherlands) for detection of the exon copy number changes in the SRD5A2 gene. For the MLPA data analysis, Coffalyser. Net software (https://support.mlpa. com/kb/coffalyser-net) was used. The Sanger sequencing and MLPA reaction products were analyzed on the ABI PRISM $® 3500$ Genetic Analyzer (Thermo Fisher Scientific, Waltham, MA, USA).

DNA from Patient 2 was analyzed with microarraybased comparative genomic hybridization (aCGH) analysis using Agilent Sure Print G3 Human CGH Microarray, 4 $\times 180 \mathrm{~K}$ (Agilent Technologies, Santa Clara, CA, USA). DNA from Patient 2 and the father were studied with real time-polymerase chain reaction (real time-PCR) on 7500 Fast Real-Time PCR System (Thermo Fisher Scientific) using custom designed primers and HOT FIREPolEva Green qPCR Mix Plus (Solis BioDyne, Tartu, Estonia). The pathogenicity of the identified variants was assessed according to the guidelines of the American College of Medical Genetics and Genomics (ACMG) [11].

Results of the Molecular Analyses. Sanger DNA sequencing revealed a novel, likely pathogenic c.146 C $>$ A (p.Ala49Asp) mutation in exon 1 of the SRD5A2 gene, present in a homozygous state in both affected siblings. Following criteria according to the ACMG guidelines were applicable for the classification of the c.146C $>\mathrm{A}$ as likely pathogenic: 1) extremely low frequency $(0.00000419,1$ in 238,672 alleles) in publically available gnomAD Exomes v.2.1.1 population database (https:/macarthurlab.org/2017/ 02/27/the-genome-aggregation-database-gnomad/) [PM2]; 2) detected in trans with a pathogenic variant [PM3];3) cosegregation with disease in two affected family members [PP1]; 4) missense variant in a gene that has a low rate of benign missense variation and in which missense variants are a common mechanism of disease [PP2], and 5) the patients' phenotypes are highly specific for a disease with a single genetic etiology (5-ARD deficiency due to pathogenic SRD5A2 mutations) [PP4].

Unexpectedly, the sequencing analysis of the parents showed that only the mother was a carrier of the c.146C $>\mathrm{A}$ mutation. The father carried a benign c. $145 \mathrm{G}>\mathrm{A}$ mutation in a homozygous state [benign interpretation in ClinVar (https://www.ncbi.nlm.nih.gov/clinvar/) and with benign classification according to ACMG criteria] [Figure 2(A)]. These findings could be explained either by non paternity or by the presence of a heterozygous deletion involving exon 1 of the SRD 5A2 gene in the father and both affected siblings. Indeed, the MLPA analysis revealed a deletion involving two probes, in exon 1 and intron 1 of the SRD5A2 gene in the father and affected siblings [Figure 2(B)]. Thus, the affected siblings were compound heterozygotes for c.146C $>\mathrm{A}$ and c.(?_-71)_(281+1_282-1)del mutations. No pathogenic mutation was present in the healthy sibling, who has inherited the c. $145 \mathrm{G}>\mathrm{A}$ benign mutation from the father.

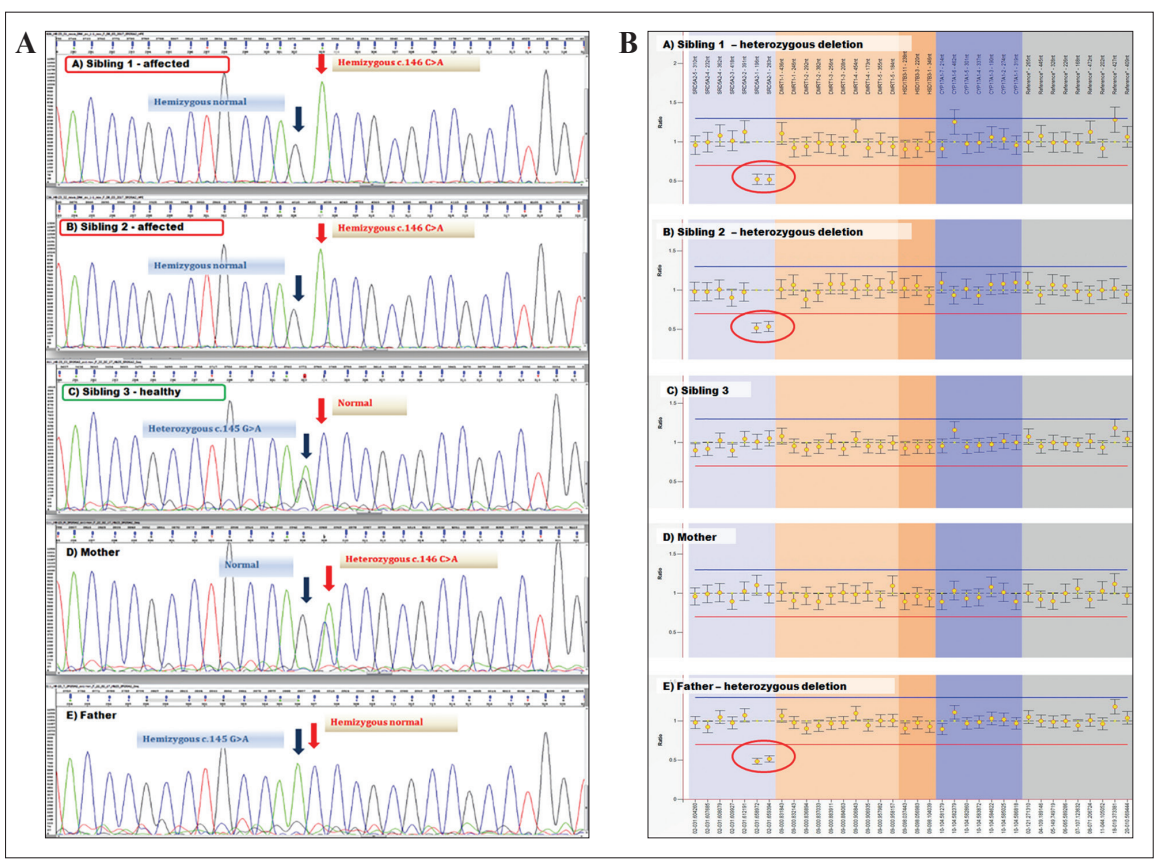

Figure 2. A) Electropherograms of Sanger sequencing analysis of SRD5A2 exon 1 in the three siblings and parents: affected siblings (Patients 1 and 2) are hemizygous for the pathogenic c. $146 \mathrm{C}>\mathrm{A}$ mutation. Healthy sibling (Patient 3) is heterozygous for the benign c. $145 \mathrm{G}>\mathrm{A}$ mutation. Mother is a carrier of the pathogenic c. $146 \mathrm{C}>\mathrm{A}$ mutation and father is hemizygous for the benign c. $145 \mathrm{G}>\mathrm{A}$.

B) Results from the MLPA analysis using P334-A3 Gonadal Development Disorder kit (MRCHolland). Two affected siblings and the father are heterozygous for the deletion in exon 1 of the gene; the healthy sibling and the mother are without aberrations in the analyzed genes. Blue and red lines represent 1.3 and 0.7 final ratio of the intensity signal, respectively. 
With the aim of more precisely defining the deletion, we performed aCGH and real-time PCR analysis. Array CGH was not successful in determination of the deletion size as a result of insufficient number of probes in the deleted region, and requirement of minimum three deleted probes for copy number variation $(\mathrm{CNV})$ calling (data not shown). Real-time PCR results revealed that five of the eight amplified fragments were amplified at half of the quantity as compared to normal control, indicating the presence of only one copy of targeted DNA sequence (het- erozygous deletion) [Figure 3(A)]. Thus, we established that the entire exon 1 and 5' untranslated region (5'UTR) of the SRD5A2 gene were deleted within the region at least approximately $8 \mathrm{~kb}$ upstream and approximately 2 $\mathrm{kb}$ downstream of exon 1 [Figure 3(B)]. The presence of $64 \mathrm{~kb}$ of interspersed repeats and low complexity DNA sequences upstream of the SRD5A2 gene did not allow for a more precise determination of the deletion breakpoints [RepeatMasker (http://www.repeatmasker.org/) track in Figure 3(B)].

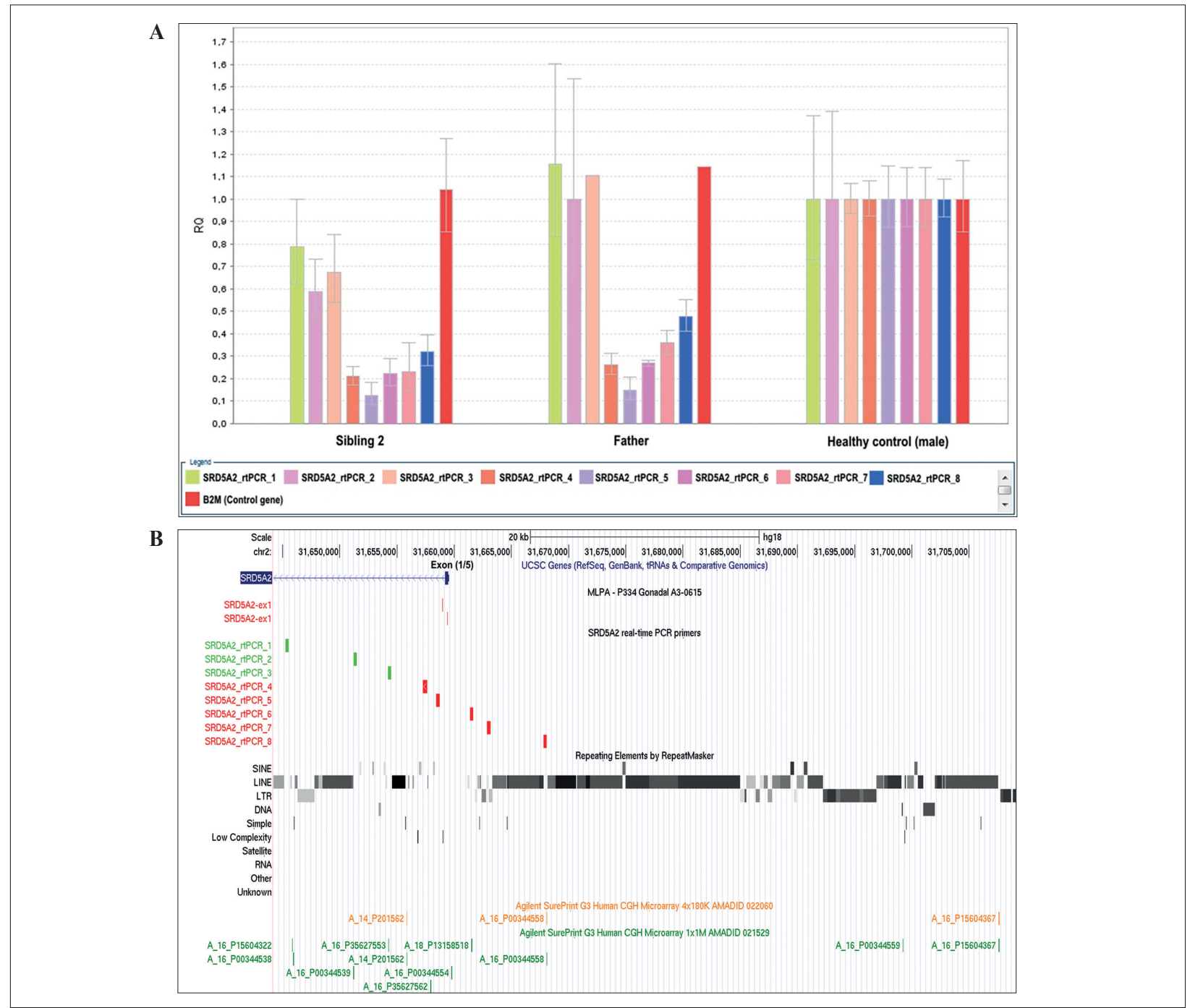

Figure 3. A) Results from the real-time PCR analysis for the approximate determination of the deletion breakpoints. The first three primers (from rtPCR_1 to rtPCR_3) showed diploid state (two copies of the targeted sequence), while the remaining five primers (from rtPCR 4 to rtPCR 8) showed haploid state (one copy of the amplified sequence) in the affected sibling and the father as compared to a normal healthy male sample used as reference. B) Graphical presentation of the targeted sequences by MLPA and realtime PCR analysis with the use of the UCSC genome browser. Besides custom tracks (MLPA-P334 Gonadal and SRD5A2 realtime PCR primers), the UCSC Genes, RepeatMasker and Agilent Arrays tracks are shown. In the custom tracks, the sequences colored red are those deleted (only one copy) in the father and the two affected siblings, while sequences colored green in the SRD5A2 realtime PCR primers track are undeleted (present in two copies). The deletion breakpoint from the 3' side (intron 1) is between SRD5A2 rtPCR 3 and SRD5A2 rtPCR 4 primers, while from the 5' side (before the SRD5A2 gene) is undetermined. There is increased complexity of the region upstream of the $S R D 5 A 2$ gene as shown by sequences annotated with RepeatMasker and lack of probes in 40 and $30 \mathrm{~kb}$ untargeted regions in the Agilent $4 \times 180 \mathrm{~K}$ and Agilent $1 \times 1 \mathrm{M}$ arrays, respectively. 


\section{DISCUSSION}

The SRD5A2 gene encodes the 5- $\alpha$-steroid reductase 2 , which is a protein (isoenzyme) that catalyzes conversion of testosterone to DHT and a very potent androgen with a crucial role in the formation of male urethra, prostate, urogenital sinus, penis and scrotum in the fetal and postnatal period. Mutations in the SRD5A2 gene result in a decreased DHT level that produces different sexual phenotypes. Depending on the DHT level, the genital phenotype can vary from normal female external genitalia, isolated hypospadias and micropenis associated with perineal hypospadias independently of the genotype $[9,11,12]$. Virilization caused by the existing testes and higher levels of testosterone, further complicates the prediction of the phenotype during puberty. Patient 1 had low grade sexual ambiguity with a slightly enlarged clitoris and palpable testes in the inguinal region, whereas Patient 2 who was first seen at the age of 7 had a similar phenotype escalating to overt male pseudohermaphroditism with a phallus of $4 \mathrm{~cm}$, perineal hypospadia, one perineal opening, and labia resembling scrota. Due to the large pubertal testes in the inguinal channel, virilization was enhanced with the enlarged Adam's apple, deep voice, and masculine muscles. In fact, Patient 2 was a natural history of the disorder that would have also been the case in Patient 1 if the early gonadectomy had not been performed.

Delayed diagnosis of 5-ARD is still common [12-14]. The XY karyotype in an individual with female or undermasculinized external genitalia should raise suspicion of 5-ARD. The measurement of DHT and testosterone/DHT ratio is not always possible, fully informative, or consistent with the diagnosis of 5-ARD $[3,15,16]$. Urinary steroid profiling using tandem mass spectrometry is proposed when available [6]. If clinical criteria for 5-ARD are met, mutational analysis is preferable for the final diagnosis and it has become increasingly available $[13,16,17]$. In Patient 1 the diagnosis was made clinically and on the basis of testosterone/DHT ratio, and in Patient 2, based on the family history and virilization during puberty. Later molecular analysis confirmed an unusual genetic constellation including a novel SRD5A2 gene deleterious mutation. Mutations causing 5-ARD are commonly located on exons 1 and 4 [3].

About 100 different changes in the SRD5A2 gene have been described, most of which are missense/nonsense loss of function mutations, including $65.0 \%$ homozygous and $35.0 \%$ compound heterozygous ones $[3,7,17]$. So far, only a few deletions have been described, without details about the borders of the deletions $[3,4,14]$. Our patients carry two changes in exon 1 of the SRD5A2 gene, one novel mis-sense mutation inherited from the mother, and a large deletion encompassing the entire exon 1 including part of the adjacent intron, inherited from the father.

To the best of our knowledge, the c.146C $>$ A mutation is novel and has not been previously described in association with 5-ARD. In the gnomAD v2.1.1 database [18], this mutation was found in a heterozygous state in only one subject of Bulgarian descent. The exact mechanism of the action of the alanine to aspartic acid substitution on the SRD5A2 protein function remains to be elucidated.

Another amino acid substitution at the same position (p.Ala49Thr) was present in a hemizygous state in the father and in a heterozygous state in the healthy sibling with apparently no effect on normal sexual development. This variant was previously described in association with hypospadias [19-21] and prostate cancer, although with inconsistent findings [22,23].

Our analysis suggested that one of the deletion breakpoints lies in intron 1, in the region located between the sequence detected with primers 3 and 4 for reverse transcription-PCR (rtPCR) [Figure 3(B)]. In this region, there are several interspersed repeats and low complexity DNA sequences, however, no such sequences are present upstream of the $S R D 5 A 2$ gene. Thus, we have no explanation of the exact mechanism of the deletion occurrence.

Although some SRD5A2 mutations cause no enzyme activity and others just influence the proportion of inactivation, no genotype/phenotype correlation has been reported, even in patients carrying the same mutations $[3,13]$. However, the clinical variability in our patients was probably due to the timing of the therapeutic procedures. If mutational analysis had been available earlier in our patients, it might have changed the sex of rearing, or made the treatment less complicated.

Sex of Rearing. Most of the patients with 5-ARD have been assigned with a female sex in the past due to the severe undervirilization of the external genitalia [24]. Sex reversal later in life can be chosen due to virilization during puberty. Those raised as females, rarely decide to change sex, well accepted in society even in the cases with a clear gender dysphoria although it requires removal of the testes, breast implantation, vaginoplasty and long-term, if not lifelong, hormonal therapy [25]. Male sex of rearing, on the other hand, helps to avoid lifelong hormonal therapy and allows the potential for fertility $[10,26]$. However, infrequent collision of the child's and/or parents' wishes, as well as the recommendations of the medical team, makes the process of decision making in these children among the most complicated issues in medicine. In Patient 1, presenting with a severe under virilization, the decision of the parents and the medical team recommendations were similar, and female sex assignment occurred early. Due to the early orchiectomy, no virilization occurred during 
puberty, however, she had to undergo breast implantation and vaginoplasty. Patient 2 was more complicated, due to the later diagnosis and underestimating of the problem by the parents until puberty, when significant virilization had occurred. In this patient many procedures such as extensive information about the therapy and outcome, psychological assessment and counseling of the child and parents, and consultations with an ethics committee were undertaken. Complicated procedures of female sex completion will be necessary in the near future. Both patients are psychologically stable and feel comfortable as females. Keeping in mind the responsibility of these decisions, the recent guidelines should be followed in 46,XY under virilized babies, and leave the possibility for sex reversal for later in life, if needed [27]. Timing of putative sex reversal is important, for example, 5-ARD deficiency has been confirmed in female athletes excelling in athletics, thus complicating their sports life [28].

In conclusion, we report the first family with two siblings with 5-ARD from the RN of Macedonia who were molecularly analyzed in detail and carry a novel genotype causing the disease. Our data confirm that molecular diagnosis in under virilized XY children should be undertaken as early as possible, as it can help timely consultation with the family in order to carefully and appropriately choose the sex of rearing.

\section{ACKNOWLEDGMENTS}

We acknowledge the collaboration of the patients from the Department of Surgery Pediatric Surgery and University Clinic for Gynecology and Obstetrics (Medical Faculty, Skopje, RN Macedonia), and editing help of DrMrSci. Elena Kochova (independent consultant).

Declaration of Interest. The authors report no conflicts of interest. The authors alone are responsible for the content and writing of this article.

\section{REFERENCES}

1. Mendonca BB, Domenice S, Arnhold IJ, Costa EM. 46,XY Disorders of sex development (DSD). Clin Endo-crinol (Oxf). 2009; 70(2): 173-187.

2. Hochberg Z, Chayen R, Reiss N, Falik Z, Makler A, Munichor $\mathrm{M}$, et al. Clinical, biochemical, and genetic findings in a large pedigree of male and female patients with 5 alpha-reductase 2 deficiency. J Clin Endocrinol Metab. 1996; 81(8): 2821-2827.

3. Maimoun L, Philibert P, Cammas B, Audran F, Bouchard P, Fenichel P, et al. Phenotypical, biologi- cal, and molecular heterogeneity of $5 \alpha$-reductase deficiency: An extensive international experience of 55 patients. J Clin Endocrinol Metab. 2011; 96(2): 296-307.

4. Andersson S, Berman DM, Jenkins EP, Russell DW. Deletion of steroid $5 \alpha$-reductase 2 gene in male pseudo-hermaphroditism. Nature. 1991; 354(6349): 159-161.

5. Vilchis F, Ramos L, Mendez JP, Benavides S, Canto P, Chavez B. Molecular analysis of the SRD5A2 in 46,XY subjects with incomplete virilization: The P212R substitution of the steroid $5 \alpha$-reductase 2 may constitute an ancestral founder mutation in Mexican patients. J Androl. 2010; 31(4): 358-364.

6. Imperato-McGinley J, Zhu YS. Androgens and male physiology the syndrome of $5 \alpha$-reductase- 2 deficiency. Mol Cell Endocrinol. 2002; 198(1-2): 51-59.

7. Sasaki G, Ogata T, Ishii T, Kosaki K, Sato S, Homma $\mathrm{K}$, et al. Micropenis and the $5 \alpha$-reductase-2 (SRD5A2) gene: Mutation and V89L polymorphism analysis in 81 Japanese patients. J Clin Endocrinol Metab. 2003; 88(7): 3431-3436.

8. Thigpen AE, Davis DL, Gautier T, Imperato-McGinley J, Russell DW. Brief report: The molecular basis of steroid $5 \alpha$-reductase deficiency in a large Dominican kindred. N Engl J Med. 1992; 327(17): 12161219.

9. Alswailem MM, Alzahrani OS, Alghofaili L, Qasem E, Almohanaa M, Alsagheir A, et al. Molecular genetics and phenotype/genotype correlation of 5- $\alpha$ reductase deficiency in a highly consanguineous population. Endocrine. 2019; 63(2): 361-368.

10. M Selveindran N, Syed Zakaria SZ, Jalaludin MY, Rasat R. Quality of life in children with disorders of sex development. Horm Res Paediatr. 2017; 88(5): 324-330.

11. Richards S, Aziz N, Bale S, Bick D, Das S, GastierFoster J, et al. Standards and guidelines for the interpretation of sequence variants: A joint consensus recommendation of the American College of Medical Genetics and Genomics and the Association for Molecular Pathology. Genet Med. 2015; 17(5): 405-424.

12. Nicoletti A, Baldazzi L, Balsamo A, Barp L, Pirazzoli $\mathrm{P}$, Gennari $\mathrm{M}$, et al. SRD5A2 gene analysis in an Italian population of under-masculinized $46, \mathrm{XY}$ subjects. Clin Endocrinol (Oxf). 2005; 63(4): 375-380.

13. Avendano A, Paradisi I, Cammarata-Scalisi F, Callea M. 5- $\alpha$-Reductase type 2 deficiency: Is there a genotype-phenotype correlation? A review. Hormones (Athens). 2018; 17(2): 197-204. 
14. Andonova S, Robeva R, Vazharova R, Ledig S, Grozdanova L, Stefanova E, et al. New territory for an old disease: 5 - $\alpha$-reductase type 2 deficiency in Bulgaria. Sex Dev. 2017; 11(1): 21-28.

15. Perry RJ, Novikova E, Wallace AM, Donaldson MD. Pitfalls in the diagnosis of $5 \alpha$-reductase type 2 deficiency during early infancy. Horm Res Paediatr. 2011; 75(5): 380-382.

16. Chan AO, But BW, Lee CY, Lam YY, Ng KL, Tung JY, et al. Diagnosis of $5 \alpha$-reductase 2 deficiency: Is measurement of dihydrotestosterone essential? Clin Chem. 2013; 59(5): 798-806.

17. Hackel C, Oliveira LE, Ferraz LF, Tonini MM, Silva $\mathrm{DN}$, Toralles MB, et al. New mutations, hotspots, and founder effects in Brazilian patients with steroid $5 \alpha$-reductase deficiency type 2. J Mol Med (Berl). 2005; 83(7): 569-576.

18. Karczewski K, Francioli L. The genome Aggregation Database (gnomAD). MacArthur Lab. 2017. (https:// macarthurlab.org/2017/02/27/the-genomeaggregation- database-gnomad/.)

19. Silveri RI, Russell DW. $5 \alpha$-Reductase type 2 mutations are present in some boys with isolated hypospadias. J Urol. 1999; 162(3): 1142-1145.

20. Rahimi M, Ghanbari M, Fazeli Z, Rouzrokh M, Omrani S, Mirfakhraie R, et al. Association of SRD5A2 gene mutations with risk of hypospadias in the Iranian population. J Endocrinol Invest. 2017; 40(4): 391-396.

21. Samtani R, Bajpai M, Ghosh P, Saraswathy K. SRD5A2 gene mutations - A population-based review. Pediatr Endocrinol Rev. 2010; 8(1): 34-40.

22. Fang C, Guo ZQ, Chen XY, Liu TZ, Zeng XT, Wang XH. Relationship between SRD5A2 rs9282858 poly- morphism and the susceptibility of prostate cancer: A meta-analysis based on 20 publications. Medicine (Baltimore). 2017; 96(19): e6791.

23. Pearce CL, van den Berg DJ, Makridakis N, Reichardt JK, Ross RK, Pike MC, et al. No association between the SRD5A2 gene A49T missense variant and prostate cancer risk: Lessons learned. Hum Mol Genet. 2008; 17(16): 2456-2461.

24. Costa EM, Domenice S, Sircili MH, Inacio M, Mendonca BB. DSD due to $5 \alpha$-reductase 2 deficiency From diagnosis to long term outcome. Semin Reprod Med. 2012; 30(5): 427-431

25. Byers HM, Mohnach LH, Fechner PY, Chen M, Thomas IH, Ramsdell LA, et al. Unexpected ethical dilemmas in sex assignment in 46,XY DSD due to 5 - $\alpha$ reductase type 2 deficiency. Am J Med Genet C Semin Med Genet. 2017; 175(2): 260-267.

26. Houk CP, Lee PA. Consensus statement on terminology and management: Disorders of sex development. Sex Dev. 2008; 2(4-5): 172-180.

27. Wisniewski AB, Mazur T. 46,XY DSD with female or ambiguous external genitalia at birth due to androgen insensitivity syndrome, $5 \alpha$-reductase- 2 deficiency, or $17 \beta$-hydroxysteroid dehydrogenase deficiency: A review of quality of life outcomes. Int J Pediatr Endocrinol. 2009; 2009: 567430.

28. Fenichel P, Paris F, Philibert P, Hieronimus S, Gaspari L, Kurzenne JY, et al. Molecular diagnosis of $5 \alpha$-reductase deficiency in 4 elite young female athletes through hormonal screening for hyperandrogenism. J Clin Endocrinol Metab. 2013; 98(6): E1055-E1059. 
